\title{
Reconstruction of land-to-sea radionuclide flux by POSEIDON/RODOS model chain: The Thermaikos Gulf base
}

\author{
A. Popov, N. Catsaros ${ }^{1}$, V. Maderich ${ }^{2}$ and S. Yushenko ${ }^{2}$ \\ OINPE, Studgorodok 1, Kaluga Region, Obninsk 249020, Russia \\ ${ }^{1}$ NCRS “Demokritos", Greece \\ ${ }^{2}$ IPMMS, Ukraine
}

\begin{abstract}
The model chain simulating the radionuclide transport in watersheds, rivers and sea waters, developed in the frame of the EC project POSEIDON, was applied to River Axios watershed and Thermaikos Gulf of Northern Aegean Sea. The model system included coupled deterministic box models for radionuclide transport on watershed (RETRACE-3), complemented by a 3D hydrodynamic model (THREETOX) and a stochastic weather generator. The generic representation of the complex watershed hydrology was used under conditions of important uncertainties in the input data. A Monte-Carlo approach was applied to tune the wash-off model. The simulated results are in reasonable agreement with scarce measurements. The results obtained demonstrate strong seasonal variability of the land-to-sea radionuclide flux and transport in the Thermaikos Gulf. Estimations of the land-to-sea radionuclide flux and the radionuclide concentration in seawater for the next 50 years were obtained using the generated weather parameter's sets. These estimates could be used to assess the radio-ecological doses and risk under worst, average and favourable climatic conditions.
\end{abstract}

\section{INTRODUCTION}

The model chain "POSEIDON-R" simulating the radionuclide transport in watersheds, rivers and seawaters, was developed in the frame of the EC project POSEIDON (IC15-CT98-0210) and was applied to River Axios watershed and Thermaikos Gulf of Northern Aegean Sea [1]. The overall goals of POSEIDON project were (1) the improvement of available models for forecasting marine radioactivity caused by accidental releases from coastal areas and (2) model's implementation into the Hydrological Model Chain of the EU Decision Support system RODOS [1].

The main aim of the Axios case study (Greece) was to evaluate the applicability of radionuclide land-to-sea transport RETRACE model along with 3D-model THREETOX for the simulation of radioactive contamination transport on the middle-size watershed actually polluted due to the Chernobyl accident. It should be noted that for the Axios case study the "long-term" version of the RETRACE model (named RETRACE-3) was used.

\section{MODEL DESCRIPTIONS}

\subsection{RETRACE model}

The following circumstances were taken into consideration while RETRACE model was developed:

- Essential spatial variability of watershed contamination along with spatial variability of runoff formation depending on the elevation and the hydrological and meteorological conditions in the watersheds;

- Essential temporal variability of runoff formation depending on the meteorological conditions on watersheds;

- Strong connection between water erosion process and wash-off process on the watersheds for many important radionuclides including radio-caesium;

- Complex physico-chemical transformation processes of radionuclides in soil and inland waters.

Taking into consideration the conditions listed RETRACE model design has the following features: 
- The whole watershed territory is divided in several sub-territories (sub-catchments), which differ in hydrological and/or contamination characteristics;

- Each sub-territory is simulated by the same lumped wash-off model named Watershed Runoff Module (WMR) [3]; therefore RETRACE model module could operate with several WMR modules consequently;

- WMR is the basic block of the whole RETRACE model; it operates on daily time basis, includes lumped hydrological, soil erosion and radionuclide transport sub-models; the WMR's hydrological sub-model belongs to the class of "completed hydrological" models, e.g. it could operate during the whole year rather than during a single hydrological event; the process of runoff formation on the hill slopes is considered using a concept of water storage system [3] corresponding to different types of runoff (e.g. overland runoff, fast ground flow, deep ground flow, etc.); the evapotranspiration is simulated using the Linacre modifications of Penman-Monteith equation [5]; the soil erosion sub-model is based on empirical relationships, which could be tuned based on local (regional) measurements [3];

- WMR's radionuclide transport sub-model considers the radionuclide transformation in soil (up to four different radionuclide forms), vertical migration using of analytical solution of diffusion equation, as well as surface and sub-surface wash-out as a parts of activity balance [3];

- In the frame of RETRACE model, the WMR module gets the meteorological input data (daily precipitation depth, daily mean air temperature and relative humidity) from either historical databases or from Weather Generator Module (WGEN) [2] included in the RETRACE model;

- WMR provides output data for a single point, i.e. sub-catchment outlet; the output data are water discharge, sediment and radionuclide concentrations in water;

- WMR output could be directed either to the sea module (i.e. outside RETRACE module) or to the RETRACE Reservoir Module (RRM);

- $\quad$ RRM is a box model intended for simulation of the radionuclide transport in a river branch or in a lake; it is based on the water and suspended sediment mass balance along with radionuclide activity in water and bottom sediment conservation equations; the approach used is close to the methodology described in Ref. [6].

\subsection{THREETOX modeI}

The 3-D model THREETOX that includes modules of hydrodynamics, sediment transport and radionuclide transport was developed in IMMSP [7] to simulate the radionuclide fate in deep stratified water bodies. The hydrodynamics of THREETOX model is based on the three-dimensional, timedependent, free surface primitive equation POM model. The prognostic variables of the hydrodynamics. code are the three components of the velocity, temperature, salinity and surface elevation fields. All particulate matter is represented by three different grain size fractions. The effect of sediment on the hydrodynamics is neglected. Suspended sediment transport is described by the advection-diffusion equations, taking into account the settling velocity of the sediment grains. The vertical flux of suspended sediments at the bottom is equal to the difference between the resuspension and sedimentation rate.

The sub-model of radionuclide transport describes the specific water-sediment sorption processes. It includes the advection-diffusion equations for dissolved and adsorbed by suspended sediment radioactivity in the water column, and the equations for concentration of the dissolved and adsorbed radioactivity in the bottom deposits. The exchanges between these different phases are described.by diffusion, sorption, and sedimentation-resuspension processes. Adsorption and desorption of radionuclides between liquid and solid phases are described by the radionuclide exchange rates and by the distribution coefficients.

\section{DATA DESCRIPTION}

Excellent geographical description of River Axios watershed and Thermaikos Gulf is given in the Ref. [4]; that is why only several key parameters of the Axios coastal system arc presentcd bclow. 
River Axios watershed is located between 21-24 degrees of eastern longitude and 39-43 degrees of northern latitude, so that the watershed area is $23747 \mathrm{~km} 2$. The lower part of the watershed belongs to Greece (Hellenic Republic), while the upper and the bigger part belongs to FYROM (Former Yugoslavia Republic Of Macedonia). In general, the watershed territory is mountainous and semi-mountainous, with differences in altitude of more than 2800 meters. Such differences in altitude result in high difference in air temperatures: from $-45 \mathrm{C}$ in winter up to $+30 \mathrm{C}$ in summer. The mean annual temperature is $14.5^{\circ} \mathrm{C}$. The climate is changing from "Continental" in the mountainous part to "Mediterranean" in the deltaic plain.

Historical daily meteorological data on the region were obtained from the public domain NCEP Reanalysis data base provided by the NOAA-CIRES Climate Diagnostics Centre (Boulder, Colorado, US) from their Web site at http://www.cdc.noaa.gov/. These re-analysis data are available at grid points with $1.5-2.5$ degrees step (in both directions) for different meteorological variables. Therefore only two points of that grid are suitable for the Axios case study, namely Point $0(40.95 \mathrm{~N} 20.5 \mathrm{~W}$ ) and Point 1 $(42.85 \mathrm{~N} 20.5 \mathrm{~W})$. Point 0 is located on the southern (Greek) part of watershed, whereas Pont 1 is located close to the north boundary (FYROM territory).

Data on River Axios mean monthly water discharge was collected in the frame of the EC project MAST [8]. Additionally, there were daily discharge data available for 1994 [8]. Though Axios River is in the list of 1000 world rivers with the highest discharge, the specific feature of discharge time set is the presence of rather long periods with very low and even zero discharge in the river outlet (e.g. zero discharge in 1994 was recorded for about two months [8]). This behaviour is likely connected with important role of snow melting water income, as well as operation of several controlled reservoirs in Greece and FYROM, where waters are stored and used for irrigation. Unfortunately, there is no information on the irrigation policy and even on geometric sizes of water reservoirs that makes very difficult to simulate correctly the water balance in the river. It should be stressed that such lack of hydrological information makes the application of any river model, which is more complex than lumped models, highly questionable. It should be noted also that even mean annual discharge of Axios River is known with rather large uncertainty. So, according to Poulos et al. [4] the mean annual value is $158 \mathrm{~m}^{3} / \mathrm{s}$ whereas this parameter was estimated to be 135 or $146 \mathrm{~m}^{3} / \mathrm{s}$ in Ref. [9].

There is no data found on the River Axios water turbidity except the estimate of the mean total sediment yield $1220 \mathrm{t} /\left(\mathrm{km}^{2} \square\right.$ year) [4]. Using this estimate and mean annual discharge one could estimate mean turbidity as $5.8 \mathrm{~kg} / \mathrm{m}^{3}\left(6.8 \mathrm{~kg} / \mathrm{m}^{3}\right.$ using discharge data from [9]).

Data on surface contamination by radio-caesium for the Greek territory of the Axios watershed are available in the "Atlas" [10] whereas no published data for FYROM territory were found. The surface concentration of radio-caesium on the most part of the Greek territory varies from 10 to $30 \mathrm{KBq} / \mathrm{m}^{2}$. It was supposed that the more mountainous FYROM territory is more contaminated than the more plain Greek part of Axios watershed. Thus, the mean surface concentration of radio-caesium was supposed to be $10 \mathrm{KBq} / \mathrm{m}^{2}$ for Greek territory and $25 \mathrm{KBq} / \mathrm{m}^{2}$ for the FYROM territory.

There exist data of Greek monitoring on total $\square$-activity of Axios River water [11] since 1980 up to 1994 but unfortunately the measurements were interrupted in 1984 and re-started in 1988. So the most affected period was kept unobserved. The data available demonstrate significant contamination before Chernobyl accident. This contamination could be caused by mining activity on the FYROM territory. Another circumstance is that monitoring point is located just after the FYROM-Greece boundary that means the data represents mainly radionuclide runoff on the upper part of Axios watershed.

Resuming the data availability it should be concluded that data amount and quality do not allow to test the model but it seems possible to use the data collection for reconstruction and estimates of radioactive fluxes and doses of radiation.

\section{SIMULATION RESULTS}

Two different spatial representations of the watershed were developed and tested. As shown in Fig. 1, the first configuration (a) was the simplest one - one WRM, corresponding to the whole watershed, produces output to one RRM, corresponding to the reservoir on the Greek territory. The reservoir parameters were 
obtained by stochastic simulation using the hydrological data available. Meteorological data were used only for the Point 0 . Some simple algorithms to represent irrigation policy were developed and operated effectively [1].

(a)

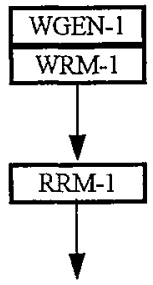

Figure 1. Spatial representation of the watershed.
In general, the results achieved have demonstrated reasonable agreement with available measurements [1] that means the applicability of the RETRACE model approach used. It was found also that some discharge peaks are originated from snow melting on the northern part of watershed, which was not described by data for Point 0 . Such discrepancy became the reason to change the spatial representation of the watershed (configuration $b$ on Fig. 1, where WRM-1 corresponds to southern, Greek part of watershed, and WRM-2

corresponds to the northern part - FYROM).

In the second configuration the watershed model was able to accept meteorological input for both

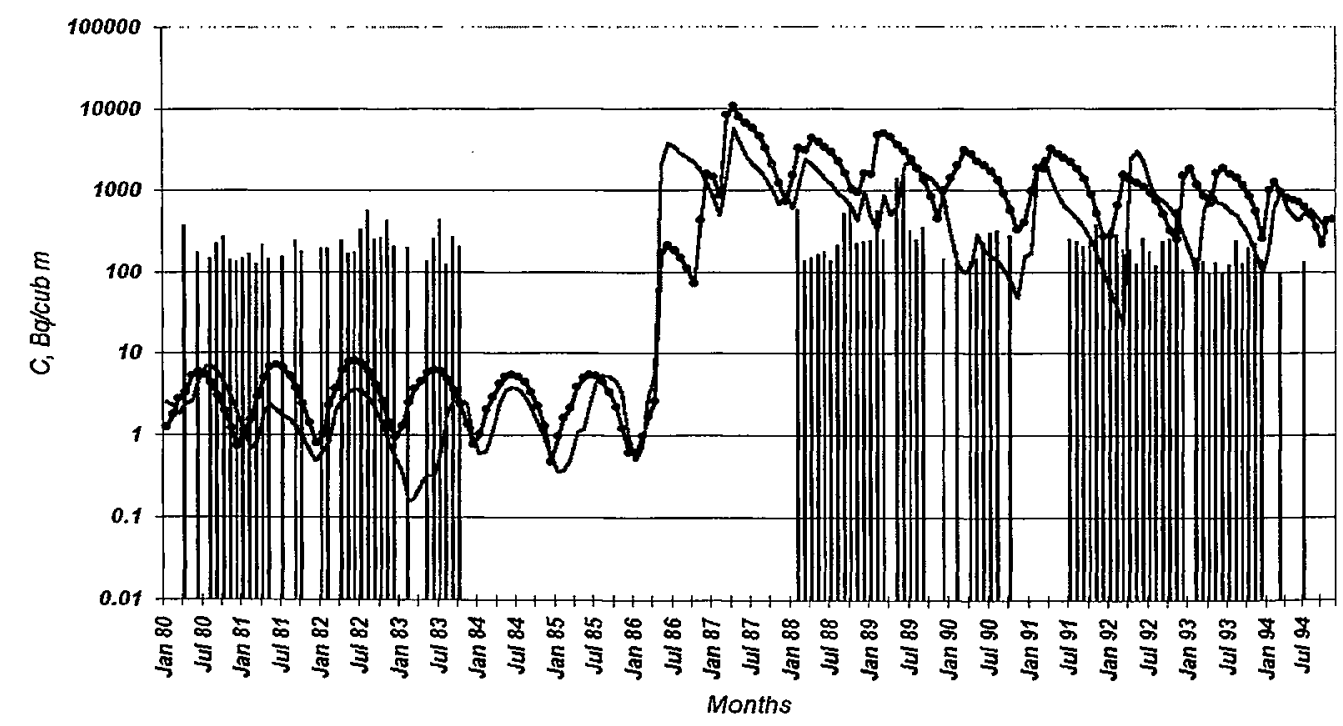

Figure 2. Mean monthly Cs-137 concentration in River Axios water: measured (bars), simulated with meteorological reanalysis data (curve without markers) and averaged for generated sets (curve with dots).

Point 0 and Point 1 , which promised to correct the disadvantages mentioned above. The single RRM represents the whole Axios River - without explicit consideration of controlled reservoirs. It was supposed that before Chernobyl accident there were traceable contamination of bottom sediments in river and reservoirs but the model were not tuned to approximate initial contamination. Simulations were made with data of meteorological re-analysis as well as with data generated by WGEN. The main results are the following:

- In spite of uncertainties and discrepancies present in input and observed data it was possible to get satisfactory simulation results, which approximate observations; 


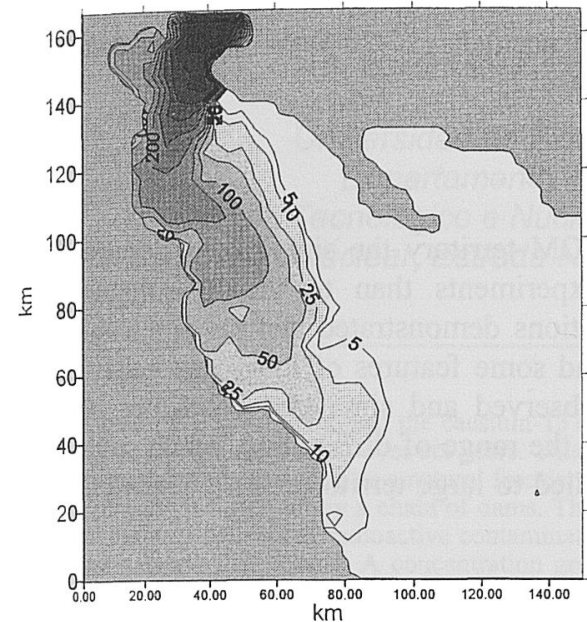

Figure 3. Input of Axios River in concentration of radiocaesium at surface in June $1987(\mathrm{~Bq} / \mathrm{m} 3)$

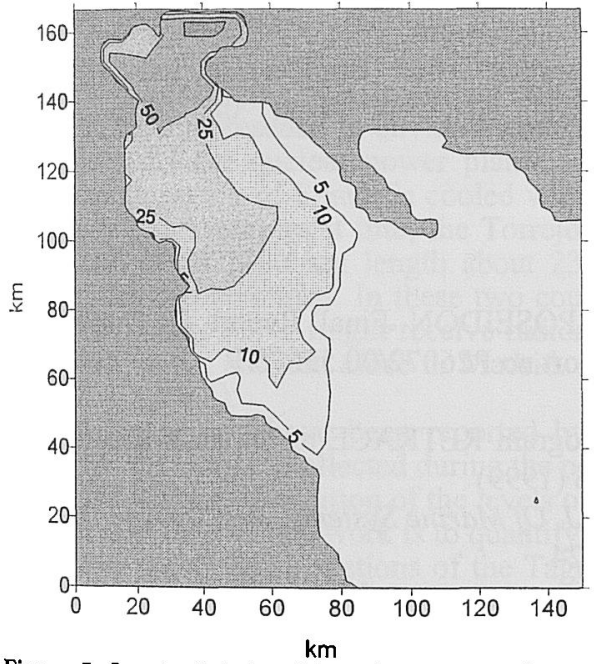

Figure 5. Input of Axios River in concentration of radiocaesium at surface in June $1988\left(\mathrm{~Bq} / \mathrm{m}^{3}\right)$

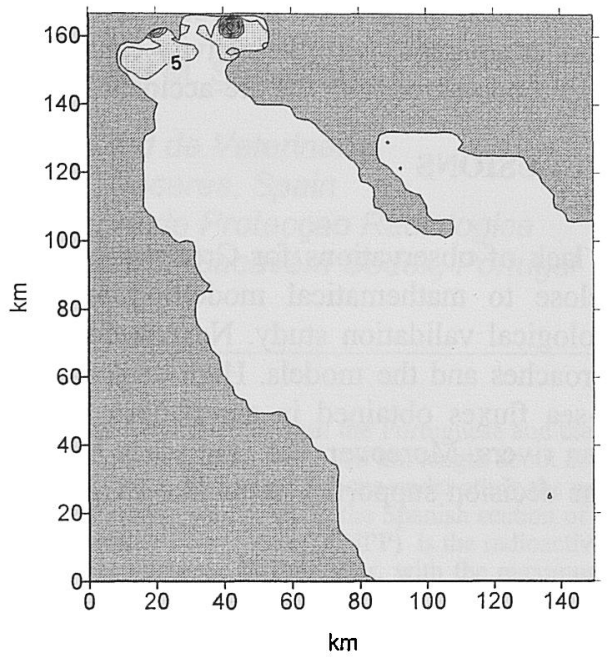

Figure 4. Input of Axios River in concentration of radiocaesium in bottom in June $1987(\mathrm{~Bq} / \mathrm{kg})$

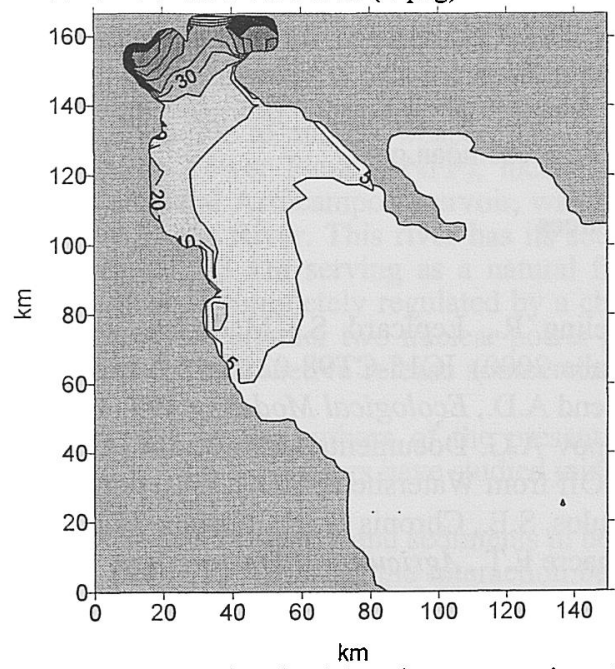

Figure 6. Input of Axios River in concentration of radiocaesium in bottom in June $1988(\mathrm{~Bq} / \mathrm{kg})$

- There exist strong seasonal variability of simulated radionuclide concentration and land-to-sea flux caused by runoff seasonal changes in the watershed (see Fig.2);

- Due to meteorological situation in 1986 , the simulated concentration of radio-caesium is about ten times more than under average conditions;

- Long-time behaviour of radio-caesium concentration in river water is defined by both decreasing of radionuclide wash-off from watershed along with processes of interaction with bottom sediment; one could determine two-exponent time dependence as observed in other European rivers, contaminated by the Chernobyl accident.

The calculations of circulation and radionuclide transport and fate in Thermaikos Bay were carried out by THREETOX for 1985-1990, using observed meteorological forcing and climatic fields of Aegean Sea temperature and salinity on liquid boundaries. The RETRACE model output (discharge and ${ }^{137} \mathrm{Cs}$ concentration) was used as input in Thermaikos Bay simulation. Because of lack of measurements, the effects of Chernobyl deposition on the sea surface and influx of the Black Sea waters through the Dardanelles have not been simulated. Therefore the results presented below demonstrated only the effect of radionuclide inflow from Axios basin. The simulation results for the ${ }^{137} \mathrm{Cs}$ concentration in near 
surface water and in the bottom deposits are given in Figures 3-6. It was shown that activity of incoming water was comparable with the activity caused by Chernobyl accident $\left(10-20 \mathrm{~Bq} / \mathrm{m}^{3}\right)$. These values are quite high if compared with the pre-accident level of $2.6 \mathrm{~Bq} / \mathrm{m}^{3}$ [12].

\section{CONCLUSIONS}

Due to lack of observations for Greek and especially FYROM territory the simulations described are more close to mathematical modelling and numerical experiments than to the hydrological and radioecological validation study. Nevertheless, the investigations demonstrated general applicability of the approaches and the models. High seasonal variability and some features of long-time behaviour of land-to-sea fluxes obtained in simulations correlate with observed and simulated behaviour of other European rivers. Moreover, the case study has demonstrated the range of difficulties, which could arise when the decision support systems like RODOS will be applied to large territories with low monitoring level.

\section{Acknowledgments}

The authors are grateful to all colleagues for the help in getting and accomplishing a NATO Fellowship Program grant, and especially to Greek colleagues from the National Centre for Scientific Research "DEMOKRITOS", Greece, for the help in data collection and discussions.

The authors are also acknowledged to the Climate Diagnostics Center (Boulder, Colorado, US) staff, which made it possible to use the NCEP Reanalysis database from the Web site at http://www.cdc.noaa.gov/

\section{References}

[1] Heling R., Lepicard S., Maderich V., et al. 2000. POSEIDON Final Report (1 October- 30 September2000) IC15-CT98-0210. 15 November 2000. Report no P26072/00.55075/P

[2] Friend A.D., Ecological Modeling 109 (1998) 121-140

[3] Popov A.G. Documentation of the RODOS External Program RETRACE to Simulate Radionuclide Wash-Off from Watersheds. RODOS Report WG4-TN99_16 (1999)

[4] Poulos, S.E., Chronis G. Th., Collins M.B., Lykousis V., J. Of Marine Systems, 25 (2000) 47-76.

[5] Linacre E.T., Agricultural Meteorology, 18 (1977) 409-424.

[6] Roshydromet. Methodology of contaminated waterbodies prognoses under conditions of NPP normal operation breaking (State Committee on Hydrology and Meteorology, Moscow, 1988)

[7] Margvelashvili N., Maderich V. and Zheleznyak M., Radiation Protection Dosimetry, 73, (1997).

[8] Krestenitis Y., Barbopoulos K. and Kourafalou V. METRO-MED, MAST-III Eloise Project, $2^{\text {nd }}$ Annual Scientific Report, NCMR, Vol. I, pp. 209-216 (1998)

[9] Perry G. D., Duffy P. B. and Miller N. L. An extended data set of river discharges for validation of general circulation models. Paper number 96JD00932 (American Geophysical Union, 1996)

[10] European Commission, Atlas of the cesium contamination of Europe after the Chernobyl accident, EUR 16733 (1998)

[11] Kritidis P. et al. Reports on environmental radioactivity monitoring in Greece. Environmental Radioactivity Laboratory/ INT-RP/NCSR"D", Aghia Paraskevi, Attiki - Greece (1994)

[12] Florou H., Kritidis P. Radiochimica Acta 66/67 (1994) 415-417 